\title{
A Study on Translation Strategies of Liyuan Opera from the Perspective of Functional Translation Theory
}

\author{
Xie Yanhong ${ }^{1, \text { a }}$ \\ ${ }^{1}$ School of Foreign Languages, Quanzhou Normal University, Quanzhou Fujian, 362000, China \\ a937090013@qq.com
}

\begin{abstract}
Keywords: Functional translation theory, Liyuan Opera, English translation.
Abstract. As one of the first National Intangible Cultural Heritages, Liyuan Opera enjoys the fame as "the Living Fossil of Ancient Southern Operas" and has the inheritance for thousands of years. Today with the idea of "Chinese culture going out", Liyuan Opera English translation still has a long way to go. In the framework of "communicative equivalence" in functional translation theory, the translators should employ the corresponding translation strategies according to the purpose of translation, to reflect the rich language style, cultural connotation and ideological background of Liyuan Opera, so that people all over the world can enjoy the unique charm of it.
\end{abstract}

\section{Introduction}

With the reputation of "the Living Fossil of Ancient Southern Operas", Liyuan Opera was originated in Quanzhou since the Song and Yuan dynasties. It was widely spread in Quanzhou, Xiamen, Zhangzhou, Guangdong, Hong Kong, Macao areas and other overseas Chinese residences in southeast Asian countries. After thousands of heritage, the plays, music singing and performance regulation in Liyuan Opera are well preserved since the Song and Yuan Dynasties, and it still enjoys the wide spread space nowadays. In the year of 2006, Liyuan Opera was approved by the Chinese State Council as one of the first National Intangible Cultural Heritage List. However, in the impact of various new media in the modern society, the prospect of Liyuan Opera is not optimistic. Compared with other Chinese operas, the development of Liyuan Opera is not smooth at all, foreign visitors lack the appropriate opportunities and channels to enjoy the beauty of Liyuan Opera. In order to improve the overseas spread, the English translation of Liyuan Opera is particularly significant.

Reviewing the researches of the domestic and international study of operas and the translation, the scholars' researches mostly focus on the traditional fields, such as the originalities, paradigms, scripts, schools and so on (Wang Wei: 2012, Wang Xiaomei: 2013, Huang Wenjuan: 2014), and the inheritance, spread and development of Liyuan Opera (Song Yan: 2014, Zhu Titi:2016), but few scholars study the translation of Liyuan Opera. Chen Fangrong points out: "As a special form of foreign propaganda translation, (the translation of Intangible Cultural Heritage operas) is not simply a Chinese-English translation, but a kind of translation variety after the translator fully digests and absorbs the of the original version of Intangible Cultural Heritage operas, and is a kind of combination of translation and introduction (Chen Fangrong:2013).” Thus, this article intends to explore the translation strategies of contemporary Liyuan Opera from the perspective of functional translation theory.

Functional Translation Theory and Communicative Equivalence

Functional translation theory was originated in Germany in the 1970s, mainly includes the Textual Theory of Katharina Reiss, the Skopos Theory of Hans Vermeer, and the "Communicative equivalence" Principle of Kristiane Nord. Functional translation theory holds that translation is not only a kind of behavioral activity of mankind, but also a kind of communicative behavior which should always take the purpose of the readers into consideration. The primary principle of the translation is the purpose of translation. Because of the differences of languages, cultural backgrounds, the ways of thinking and expression habits, there are definitely some differences between the intention and textual format of the source language writers and the acceptance of the target language readers. As a kind of intercultural information communicative activity, translation should pay more attention to the communicative function of translation in the target language culture, 
put the focus on the target language and the target language readers, and therefore decide the forms the strategies and methods of translation(Nord: 2001). According to Reiss' point of view, the ideal translation should " achieve equivalence in the ideological content, language forms and communicative functions for target language and source language(Nord: 2005)." And the "Communicative equivalence" Principle of Kristiane Nord also emphasizes the subjective initiative of the translators in the process of translation (Nord: 2005).

As a kind of classic translation, the opera translation is undoubtedly very specific. It combines the characteristics of novels, poetries, prose, commentaries and so on, and also has its own particularity, namely: it has obvious historical traces and local cultural characteristics; the text mainly relies on lines and actions to shape the characters, to promote the development of the plots and to express the theme of the play scripts. As an ancient and mature local opera, Liyuan opera not only combines many elements of traditional Chinese culture and local cultural characteristics in southern Fujian areas, but also maintains its pure opera characteristics, thus giving a hard task to the translator. The translator should exploit the corresponding translation strategies according to the different purposes of translation, to reflect the rich language style, the cultural connotation and the ideology of Liyuan Opera. Undoubtedly, both the Textual Theory and the "Communicative equivalence" Principle provide feasible ideas for the translation of Liyuan Opera.

Translation Strategies of Liyuan Opera from the perspective of Functional Translation Theory

Liyuan opera has the elegant singing phonologies, graceful dancing gestures, colorful costume styles, and historical flavor (Song Yan: 2014), it also takes the advantages of Tang poetries, Song lyrics, and Yuan songs, the long and short sentences are well proportioned and filled with high musical and visual appreciation. Considering that most of the target readers do not understand this ancient opera, the translator' s primary translation purpose should be to introduce the classical works of Liyuan Opera to the readers. The translation of Liyuan Opera includes the translation of the lines, the tunes, the allusion, the rhymes, and some old stories, tales, slangs, idioms and etc. in southern Fujian areas, which is no wonder a great challenge for the translator.

Firstly, the translator should understand the ideological content of the original script. His interpretation and reproduction of the original text (or script) are indispensable and important components in translation. On the one hand, the creative understanding of Liyuan Opera requires the translator to fully understand the writing psychology of the play writer (source language writer) and his writing purpose (communicative purpose), and then creatively translate the opera on the basis of his own literal, opera and English expression abilities. On the other hand, the creative understanding and creative translation cannot deviate from the creative intention of the play writer. On the contrary, the translator should bear the purpose of realizing the interaction between the writer and target readers in mind, then starts his re-creation on the basis of being faithful to the original script, thus producing a good translation which can realize the interactive communication between the writer and the target readers. In the process of translation, the translator plays a role as a bridge between the writer and translator, the writer and target language readers, and the translator and target language readers.

Secondly, the translator should employ different translation strategies from the lingual perspective for different scripts or different parts of the same script. Liyuan opera is a combination of literary, dance, music and opera, whose audience have a unique artistic taste and appreciation habits, and are often greatly influenced by the market factors. Because of the differences in language structure and rhetorical habits between English and Chinese, the translator should consider the method of interpreting on the basis of literal translation instead of simply pursuing the direct conversion of linguistic signs and text forms; he should try to accurately interpret the underlying intention of the script, make clear of the obstacles for the target audience, show the whole play to them and help them understand the greatest artistic charm of Liyuan Opera.

In addition, the translator should be familiar with cross-cultural communication and undertake the responsibility of cultural spread. Translation is an important channel and a bridge for cultural 
communication among different countries. In the translation process, the translator is required to thoroughly understand the society and culture of his own country and that of the other country as well. Only if the translator is familiar with the two languages and related cultural information, could he realize the cross-cultural communicative function of the translation. The translation of Liyuan Opera is a kind of cross-cultural communication beyond time and space, thus the translator should always take account of the cultural implication between lines. The translator' $s$ understanding of the traditional culture is the prerequisite of his translation, which decides whether the translator can interpret the original script, whether he can translate the cultural background of the source language, and whether he is able to introduce the cultural factors of Liyuan Opera to the target audience.

An example of the translation of Liyuan Opera

In the process of Liyuan Opera translation, the translator should involve himself in the interpretation of the works according to the translation purpose, and then employ different translation strategies in a prudent and innovative way. If the target audience are common English or American people and the translation is for entertainment only, then the translator may have enough translation freedom, and the opera can be translated into the popular version. While if the target audience are cultural professionals, drama scholars and researchers, the translator should strictly base on the original script, give full play to his literal and creative ability to explain the cultural connotation and the essence of the opera to realize the communicative equivalence of Liyuan Opera translation. Since Liyuan opera contains a variety of image combinations and is full of a large number of symbols, inter-textualities, puns, metaphors and other rhetorical methods, the translation should also try to maintain its corresponding combination of image and rhetorical style. Of course, due to the great differences between Chinese and English languages and cultures, the cultural connotation of the original script is inevitably diminished, but it should not affect the overall communicative equivalence between the source script and the translation.

Following is an example of how the translator employs the translation strategies to translate Liyuan Opera, which is the translation of Chen San' s monologue in the 24th scene of "Chen San and Wu Niang"(adapted version), the most famous and popular classical play of Liyuan Opera(Zheng Guoquan: 2001).

Source script: 有缘千里终相见, 无缘至此难分离。感谢佳人真心意, 六月登楼投荔枝。望 阿娘一言为凭依, 早定佳期, 若还迁延, 我身会先死, 一点灵魂会飞在你身边。

Translation references: We are so lucky that we can finally meet again after all the traumas, and we are so unlucky that we have to depart now. Thank you for your sincere love for me, and I will never forget the love lychee you gave me from the pavilion on that afternoon in June. I wish you could believe in me and schedule our wedding as soon as possible. If there was any delay or incident, I would devote my life, and my soul will always be with you.

Analysis: The lines are adapted from Chen San' s letter to Wu Niang, which express Chen San' s deep love for his lover Wu Niang, the sentences are neatly structured with rich rhythm. There are Minnan(southern Fujian areas) dialect words like "佳人、阿娘、迁延” and other cultural elements like "荔枝、佳期、灵魂” in the letter. When translating these lines, the translator should adopt the corresponding translation strategies according to different translation purposes to achieve communicative equivalence. The first sentence strictly keeps the format of the source script; the so-called "千里" is an exaggeration which means the lovers finally meet again after experiencing all the hardships; "佳人、阿娘" refer to the heroine, who is Chen San’ s lover Wu Niang, since this is the letter from Chen San to Wu Niang, "you” may be used to refer to Wu Niang; While lychee plays a critical role in the relationship between the lovers ("Chen San and Wu Niang" is also named "The Mirror and the Lychee"), the translator can add the footnote to his translation to help the target 
audience to understand the background of the play; "佳期" implies a good wedding day in the Chinese culture, the translator may not ignore the cultural information in his translation to help the target audience to understand and accept the opera.

\section{Conclusion}

As one of the first National Intangible Cultural Heritages, Liyuan Opera enjoys the fame as "the Living Fossil of Ancient Southern Opera" and has the inheritance for thousands of years. Today with the idea of "Chinese culture going out", Liyuan Opera English translation still has a long way to go. In the framework of "communicative equivalence" in functional translation theory, the translators should employ the corresponding translation strategies according to the purpose of translation, to reflect the rich language style, cultural connotation and ideological background of Liyuan Opera, so that people all over the world can enjoy the unique charm of it.

\section{References}

[1] Chen Fangrong. Translation of Cultural Diversity and Intangible Cultural Heritage, Journal of Zhejiang Normal University (Social Science Edition), 2013 (3): 64-69.

[2] Huang Wenjuan. On the Value Orientation of the Civic Scholars in Liyuan Opera - take The Mirror and The Lichee and Mr. Dong \& Mrs. Lee for Example, Journal of Fujian Normal University (Philosophy and Social Sciences), (5): 99-106.

[3] Nord, Christiane. Translation as a Purposeful Activity-Functionalist Approaches Explained. Beijing: Foreign Language Teaching and Research Press, 2005.

[4]Nord, Christiane. Translation as a Purposeful Activity-Functionalist Approaches Explained. Shanghai: Shanghai Foreign Language Education Press, 2001.

[5] Song Yan. Ancient Liyuan Opera of Heritage Classics - On the heritage of the Liyuan Opera and the southern Fujian culture, Arts, 2014 (6): 85-99.

[6]Wang Wei. Transcendental Imagination: A Review of the Research Paradigm of Liyuan Opera (2000-2010), Fujian Tribun(Humanities and Social Sciences), 2012 (3): 110-113.

[7]Wang Xiaomei. Contemporary Protection and Inheritance of Liyuan Opera in the Perspective of "Intangible Cultural Heritage", Fujian Tribune (Humanities and Social Sciences Edition), 2013 (8): 145-149.

[8]Wu Jieqiu. On the Art History of Liyuan Opera, Beijing: China Opera Press, 1996: 358.362.

[9]Zheng Guoquan. The Operas and Dialects of Quanzhou in Ming and Qing Dynasties, Beijing: China Opera Press, 2001: 425-433.

[10]Zhu Titi. On the Evolution of the Vowel System in Quanzhou Dialect Since the 16th Century in the Perspective of the Rhyme of Liyuan Opera, Southwest Academic, 2016 (4): 217-223. 